\title{
Preliminary molecular evidence associating a novel BRCA1 synonymous variant with hereditary ovarian cancer syndrome
}

\author{
Angelo Minucci ${ }^{1}$, Paola Concolino ${ }^{1}$, Maria De Bonis ${ }^{1}$, Alessandra Costella ${ }^{1}$, Ida Paris ${ }^{2}$, Giovanni Scambia ${ }^{2}$ and \\ Ettore Capoluongo ${ }^{1}$
}

\begin{abstract}
Extensive molecular screening of the BRCA1/2 (BRCA) genes by massively parallel sequencing (MPS) identified variants of uncertain (or unknown) significance (VUS) and novel variants. We performed a molecular characterization of a novel BRCA1 synonymous variant discovered in a family with hereditary ovarian cancer (HOC) syndrome. We showed that the BRCA1 c.5073 A > T variant might play a pathogenic role in HOC syndrome in this family.
\end{abstract}

Pathogenic variants (PVs) in the BRCA1/2 (BRCA) genes predispose carriers to early onset hereditary breast and/or ovarian cancers (HBOCs).

A major limitation of $B R C A$ testing, especially after adoption of the massively parallel sequencing (MPS)based approach, is the increased number of uncertain (or unknown) significance(VUS) or novel variants. At the molecular level, a fraction of these variants occurs within the exon-intron boundary in highly conserved GT and AG dinucleotides at the 5'-intron or 3'-intron ends, respectively, resulting in either deletion of the exon or retention of the adjacent intron.

In this study, we report a 53-year-old Italian patient with a family history of high-grade serious ovarian cancer (onset age: 52-year-old) who was referred to our laboratory for $B R C A$ testing (Fig. 1).

The BRCA status obtained by MPS ${ }^{1}$ and MLPA (Multiplex Ligation-dependent Probe Amplification) ${ }^{2,3}$ did not reveal any known PVs. However, the patient carried a BRCA1 variant in exon 17 (c.5073 A>T; NCBI Reference

Correspondence: Angelo Minucci (angelo.minucci@virgilio.it)

(angelo.minucci@policlinicogemelli.it)

${ }^{1}$ Polo Scienze per Immagini, di Laboratorio e Infettivologiche, Teaching and Research Hospital "Agostino Gemelli" Foundation, Rome, Italy

2Department of Obstetrics and Gynecology, Division of Gynecologic Oncology,

Teaching and Research Hospital "Agostino Gemelli" Foundation, Rome, Italy
Sequence: NM_007294.3; GRCh37; Fig. 2a). We considered the $c .5073 \mathrm{~A}>T$ variant to be novel because (a) we did not identify it among more than 4000 routinely analyzed alleles and (b) it was not found in the Clin Var. (https://www.ncbi. nlm.nih.gov/clinvar/), Ensemble (http://www.ensembl.org), Human Genome Mutation (www.hgmd.cf.ac.uk), ExAC (http://exac.broadinstitute.org), or 1000G (http:// www.internationalgenome.org) databases.

In addition, family screening revealed the maternal origin of this variant and showed that the proband's brother was a healthy carrier of the $c .5073 T$ allele (Fig. 1). All subjects gave their written informed consent for $B R C A$ testing prior to blood sampling.

Several studies have shown that bioinformatics prediction tools can be used to prioritize variants for splicing assays $^{4}$. The $c .5073 A>T$ variant involves the AG dinucleotides at the 3 ' end of exon 17, and in silico analysis showed a high probability of pathogenicity due to the disruption of the WT splice donor, affecting the BRCT domain of BRCA1 (Supplementary Table 1).

For these reasons, to determine whether the $B R C A 1$ c.5073 $T$ allele impaired the normal splicing process of exon 17, we analyzed the patient's RNA. Total RNA was isolated from the patient's leukocytes using the TRIzol ${ }^{\circledR}$ protocol (Thermo Fisher Scientific, Waltham, MA, USA) and retro-transcribed using the Transcriptor High Fidelity

\section{(c) The Author(s) 2018}

(c) (i) Open Access This article is licensed under a Creative Commons Attribution 4.0 International License, which permits use, sharing, adaptation, distribution and reproduction cc) in any medium or format, as long as you give appropriate credit to the original author(s) and the source, provide a link to the Creative Commons license, and indicate if changes were made. The images or other third party material in this article are included in the article's Creative Commons license, unless indicated otherwise in a credit line to the material. If material is not included in the article's Creative Commons license and your intended use is not permitted by statutory regulation or exceeds the permitted use, you will need to obtain permission directly from the copyright holder. To view a copy of this license, visit http://creativecommons.org/licenses/by/4.0/. 


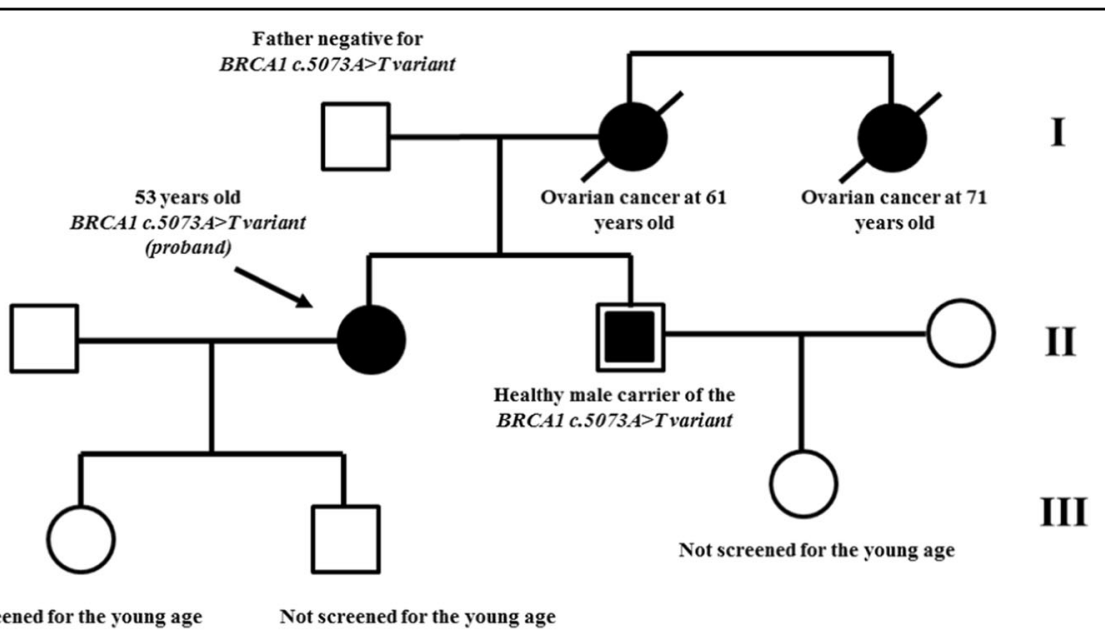

Fig. 1 The pedigree of the proband's family, cancer type, age onset, and MPS results are reported. We were unable to test other affected family members because these relatives had died. Moreover, we tested proband's father to exclude the paternal origin of the variant and proband's brother to enroll in a surveillance program for cancer prevention

cDNA Synthesis Kit (Roche Diagnostics, Basel, Switzerland). cDNA was amplified using one primer pair that was specific to BRCA1 exons 15-18 and another primer pair to demonstrate alternative splicing of exon 17. PCR was carried out using the Promega PCR Master Mix (Madison, WI, USA) with the following conditions: $95^{\circ} \mathrm{C}$ for $2 \mathrm{~min}$, $95^{\circ} \mathrm{C}$ for $30 \mathrm{~s}, 60^{\circ} \mathrm{C}$ (for the first pair) or $52^{\circ} \mathrm{C}$ (for the second pair) for $30 \mathrm{~s}$, and $75^{\circ} \mathrm{C}$ for $1 \mathrm{~min}$ for 35 cycles with a final extension incubation at $72{ }^{\circ} \mathrm{C}$ for $10 \mathrm{~min}$.

The amplified BRCA1 WT and mutated alleles were distinguished by capillary electrophoresis on an Experion ${ }^{\mathrm{Ts}}$ Automated Electrophoresis System (BioRad, Hercules, CA, USA) following the manufacturer's instructions ${ }^{5}$.

All cDNA samples showed a fragment of $499 \mathrm{bp}$, corresponding to the expected amplification product of the c.5073A allele, confirming that they were successfully retro-transcribed. Moreover, an additional band longer than the WT allele appeared in the mutated cDNAs of two carriers (the proband and her brother) due to possible alternative splicing of exon 17 (Fig. 2b). To verify this hypothesis, a second amplification was performed. Analysis of the sequencing data showed that the c.5073T allele affected splicing reactions, resulting in the retention of the 153-bp intron 17 and allowing maintenance of the reading frame and possible translation of putative BRCA1 protein 51 amino acids longer than the WT protein. However, this splicing error introduces a stop codon (TAA) $42 \mathrm{bp}$ after the $c .5073 A>T$ substitution $(c .5074+42$; Fig. 2c) terminating translation and results in a truncated protein.

Correct interpretation of the $B R C A$ variants identified during MPS molecular testing is a critical step for definitive diagnosis, risk stratification of patients, and clinical decision-making. Integration of MPS with other molecular and cellular techniques to assess the significance of
VUS or novel variants improves the sensitivity of $B R C A$ testing overall, thereby leading to even more effective personalized medicine. This family of variants may also include synonymous variants in which a change occurs in the coding region of a gene but does not alter the amino acid sequence. In cancer, $\sim 15 \%$ of synonymous variants have been estimated to cause human genetic diseases due to splicing defects $^{6}$. Most frequently, the reported mechanisms are related to dysfunctional exonic splicing regulatory sites, such as enhancers and silencers. In this context, mRNA analysis, most preferably conducted on patients' blood samples, can be used to verify the pathogenicity of these specific sequence variations.

In this study, we report the functional evaluation of a novel BRCA1 splicing variant, c.5073 $A>T$ (p.Thr1691Thr). In silico analysis predicted that this variant exerted a pathogenic effect on normal splicing of BRCA1 exon 17. Through analysis of total RNA, we demonstrated that the $c .5073 \mathrm{~T}$ allele caused an impairment of the normal splicing process of exon 17.

The results reported herein could explain the pathogenicity of this novel variant, particularly in view of the onset of ovarian cancer in this family.

In the Breast Cancer Information Core (BIC) database (https://research.nhgri.nih.gov/bic/) another variant (c.5073 $A>G$, rs80356853, p.Thr1691Thr) has been reported and classified as VUS. Based only on the variant classification criteria by ENIGMA (Evidence-based Network for the Interpretation of Germline Mutant Alleles) rather than on functional studies, it has recently been re-classified as a "likely benign variant" (Clin Var. source). It is peculiar that the c.5073 A>G (rs80356853) variant, for which bioinformatics analysis associates the same outcomes as the $c .5073$ $A>T$ variant, is a "likely benign variant". 
A

\begin{tabular}{|c|c|c|c|c|c|c|c|c|}
\hline id & type & Retseq ID & NI variant & A v variant & ESamples & dbSNP ID & VF\% & gene \\
\hline chr13:32913691:CiT & $s \cos$ & NM_000059 & CS199C>T & p.Sen1733ser & 0 & r:28897734 & 49.64 & BRCA2 \\
\hline chr13:32812299:Tic & $s_{s} \cos$ & NM_o00059 & C3807т>C & pVal1269Val & 0 & is 5443304, & 50.6 & BRCA2 \\
\hline chr1332815005:GC & $s_{s} \cos$ & NM_.000059 & $\cos 136 x$ & p.Val2171val & 0 & rs206076, & 1000 & BRCA2 \\
\hline $\operatorname{chr17741244429\mathrm {Cr}T}$ & $=\cos$ & - NM_007294 & C31196>A & p.Ser10404asn & 0 & ma986852. & 45.79 & BRCAI \\
\hline chr13:329075466::- & D.JNTRON & NM_oco0os9 & C1909-22_,1909_ & - & 0 & r:276174816, rsS87. & 34.71 & $B R C A 2$ \\
\hline Chr177:41251931:GA & SINTRON & - NM_007294 & $C A 42-3 A C>T$ & - & 0 & rs799923, & 48.59 & BRCAI \\
\hline Chr13:32929387:T:C & $5 . \cos$ & NM_.000059 & c.7397T>C & pAla2466Alo & 0 & m169547. & 99.83 & BRCA2 \\
\hline chr13332973012AC & 5.3 UTR & NM_000059 & $C 10362 A) C$ & - & 0 & rsis869, & 50.26 & BRCA2 \\
\hline chr17:41219626:TA & $s_{s} \cos$ & N NM_007294 & CS073A>T & p.Thr1691Thr & 0 & & 48.25 & BRCAI \\
\hline chr13:32913055/AG & $s_{s} \cos$ & NM_000059 & $C A S 63 A>G$ & pleu1s21leu & 0 & rs206075, & 99.51 & BRCA2 \\
\hline chr133.32906729ACC & $s \cos$ & NM.000059 & $(1114 A) C$ & PAsn372His & 0 & $\cdot 15879255323,512$ & 53.08 & $B R \mathrm{BA}_{2}$ \\
\hline
\end{tabular}

B
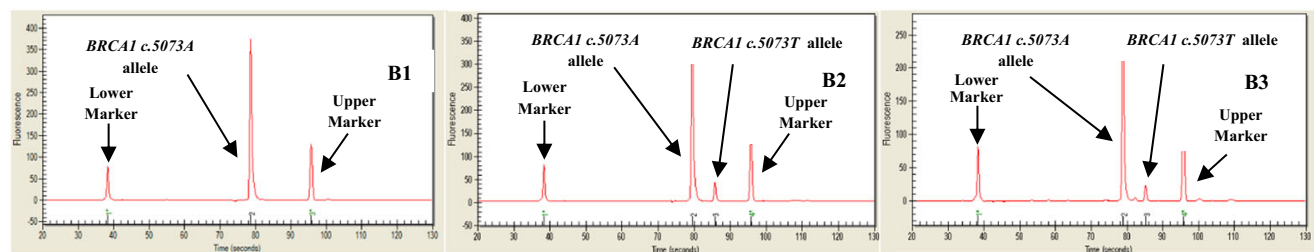

C

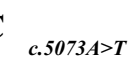

Exon 17/intron 17

Premature stop codon
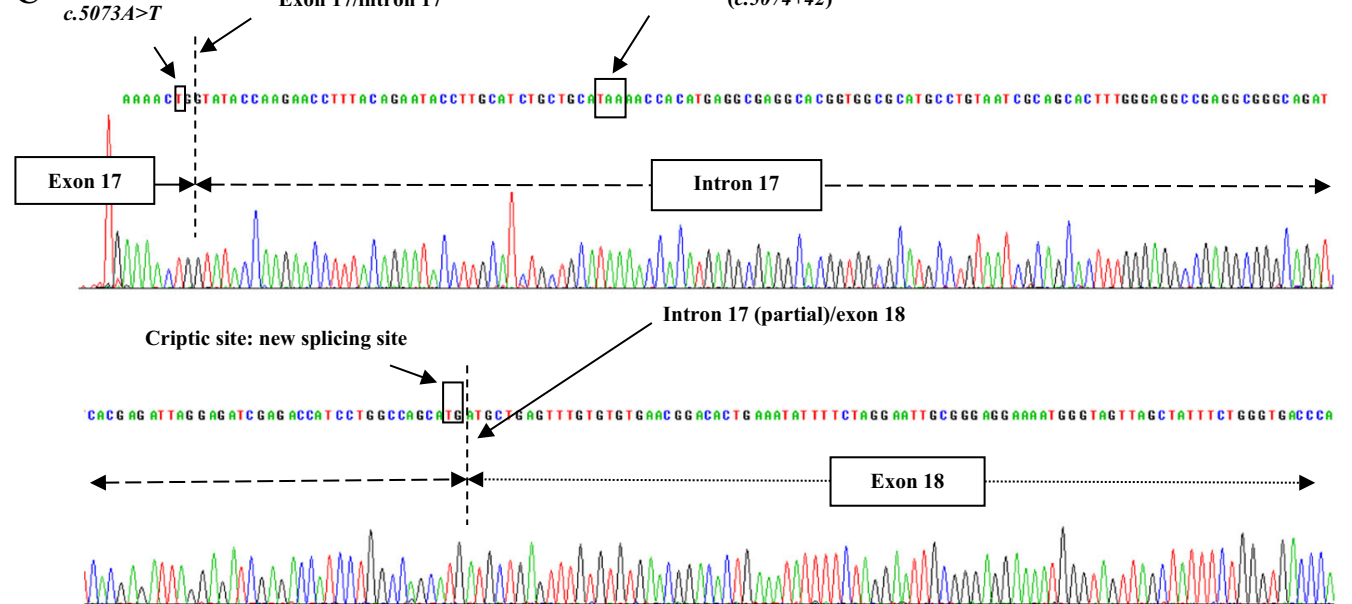

Fig. 2 a Results of the proband's genome obtained by MPS of the BRCA genes. Sequencing data, depth and variant calling were obtained by Amplicon Suite (Smart Seq, (Novara, Italy) software. b Capillary electrophoresis was used to check the amplification of the cDNAs of two women WT (b1) for the BRCA1 c.5073A>T variant, the proband (b2) and her brother (b3) carrier of the c.5073A>T variant. Arrows highlight the peaks corresponding to lower and upper markers and c.5073A $T$ alleles. In the control (b1) only the PCR product (499 bp) of the c.5073A allele is present. On the contrary, in the patient (b2) and her brother (b3), the mutated CDNAs gives two PCR products: the 499 bp band of the BRCA1 c.5073A allele and additional band of $652 \mathrm{bp}$ due to intronic retention of $153 \mathrm{bp}$ caused by the BRCA1 C.5073T allele. It is interesting to note that in both carriers, the proportion of the c.5073T allele is conserved (about 10\% of the c.5073A allele). To verify the alternative splicing of the exon 17, Sanger sequencing was used c. The c.5073T allele causes the activation of a cryptic-splicing site resulting in a partial retention of 153 bp of intron 17. Moreover, we underline that the translation of the c.5073T allele stops after $42 \mathrm{bp}$ (BRCA1 c.5074+42) due to the introduction of a stop codon (TAA codon) (as highlighted by the arrows)

However, we underline that $B R C A 1$ c.5072 $C>T$ results in aberrant splicing, whereas $c .5072 C>A$ has no effect on splicing ${ }^{7}$. We believe that further investigations are needed.

In conclusion, this case highlights the importance of studying the effects of novel $B R C A$ variants at the mRNA level to verify their potential role in disease onset and to better define the HOC risk in the proband and family members.

\section{Limitations}

It is generally accepted that variants resulting in a single major transcript lacking an open reading frame are deleterious ${ }^{8}$.

We emphasize that further evidence is needed to assign with certainty a pathogenic role to the $c .5073 \mathrm{~T}$ allele. In particular, it should be carefully evaluated whether the mutant allele causes both WT and aberrant transcripts or, alternatively, if the aberrant transcript is degraded by 
nonsense decay. In the same way, changes in the levels of low-abundance alternative splicing events could have an impact either directly or by altering the function or levels of endogenous transcripts, including full length mRNA. In addition, more evidence on the effects of the novel transcript on cellular function and the molecular interaction of WT BRCA1 with other associated genes is necessary. It will also be important to extend this investigation to ovarian tissue to gain a broader understanding of this splicing variant.

However, we believe that the use of cDNA studies is never conclusive. Multifactorial likelihood analysis methods that combine bioinformatics, pathologic, and clinical information are needed to definitively classify this type of variants as pathogenic or benign.

In conclusion, although this is preliminary data, we believe it is important to report these molecular evidence associated with the $B R C A 1$ c.5073A $>T$ variant.

\section{HGV Database}

The relevant data from this Data Report are hosted at the Human Genome Variation Database at https://doi.org/10.6084/m9.figshare.hgv.1933.

\section{Conflict of interest}

The authors declare that they have no conflict of interest.

\section{Publisher's note}

Springer Nature remains neutral with regard to jurisdictional claims in published maps and institutional affiliations.
Supplementary information is available for this paper at https://doi.org/ 10.1038/s41439-018-0003-0.

Received: 26 October 2017 Revised: 14 February 2018 Accepted: 8 March 2018.

Published online: 20 April 2018

\section{References}

1. Minucci, A. et al. Clinical impact on ovarian cancer patients of massive parallel sequencing for BRCA mutation detection: the experience at Gemelli hospital and a literature review. Expert Rev. Mol. Diagn. 15, 1383-1403 (2015).

2. Concolino, P. et al. Advanced tools for BRCA1/2 mutational screening: comparison between two methods for large genomic rearrangements (LGRs) detection. Clin. Chem. Lab Med. 52, 1119-1127 (2014).

3. Minucci, A. et al. Competitive PCR-high resolution melting analysis (C-PCRHRMA) for large genomic rearrangements (LGRs) detection: a new approach to assess quantitative status of BRCA1 gene in a reference laboratory. Clin. Chim. Acta 470, 83-92 (2017).

4. Vreeswijk, M. P. et al. Intronic variants in BRCA1 and BRCA2 that affect RNA splicing can be reliably selected by splice-site prediction programs. Hum. Mutat. 30, 107-114 (2009).

5. Minucci, A. et al. Identification of RFLP G6PD mutations by using microcapillary electrophoretic chips (Experion). J. Sep Sci. 31, 2694-2700 (2008).

6. Mucaki, E. J., Ainsworth, P. \& Rogan, P. K. Comprehensive prediction of mRNA splicing effects of BRCA1 and BRCA2 variants. Hum. Mutat. 32, 735-742 (2011).

7. Ahlborn, L. B. et al. Splicing analysis of 14 BRCA1 missense variants classifies nine variants as pathogenic. Breast Cancer Res. Treat. 150, 289-298 (2015).

8. Krawczak, M., Reiss, J. \& Cooper, D. N. The mutational spectrum of single basepair substitutions in mRNA splice junctions of human genes: causes and consequences. Hum. Genet. 90, 41-54 (1992). 